\title{
A crystal ball made of agents
}

\author{
Dr. José Miguel Castillo \\ Tecnalia. Madrid, Spain
}

\begin{abstract}
This article presents an agent-based solution to model the opinions of an experts group with the aim of predicting possible future scenarios.

The need to envision the future is not new; it has existed since the beginning of human-kind. What it is new is the applicable technology that is available in a specific period of time.

It is not usual to find a critical social system which evolves according to predictable guidelines or tendencies. Because of that reason, technical prediction based on past and present data is not reliable.

This paper includes the process description of eliciting information from a group of experts and a real case study.
\end{abstract}

Keywords - MAS, Prevention, Prospective, Scenarios, Foresight.

\section{INTRODUCTION}

$\mathrm{T}$ HIS article introduces the application of the agents technology in the field of prediction. Mathematical models are useful to foresee how dynamic systems evolve. A social environment evolves as a dynamic system, with phases of stability, instability, or even worse, of a chaotic nature. However, regarding the study of future social scenarios mathematical modelization is not applicable. This field has been classically treated by Prospective [8]. Prospective studies have been usually developed by using statistical methods. The Delphi [6] and the Cross impacts [15] methods are standards in the field of Prospective.

What is new in this paper is the use of agents [10] based on Artificial Intelligence procedures, instead of statistical methods.

The method and its response are being validated with real case studies. This paper introduces a real case study that deals with the future of the Common Policy of Security and Defense in Europe in the temporal horizon of the year 2020.

\section{WAYS TO FORESEE THE FUTURE}

The necessity to foresee the future is not new. Man has always felt the necessity to predict what is going to happen. We can gather the different methods to foresee the future in

This project has been partially funded by the Spanish Ministry of Science and Innovation under grant PID-520100-2009-1. four main groups: Supernatural, Hermeneutics, Technical and Anticipatory.

Future facts or situations known by revelation, prophecy or even astrology can be included as a supernatural way to foresee the future. Unfortunately, this group does not include serious and trustable procedures, so it is not advisable to rely on such techniques.

Hermeneutics is related to social interactions focused to discuss about the future. Opinions, utopian situations and even science fiction can be included in this group.

Within the Technical group we can find mathematical models that are developed to extrapolate past and present data in order to predict future results. The study of tendencies permits us to approximate future situations in stable dynamic systems. Econometrics, demography and meteorology are sciences that can be included in this group.

Unfortunately, social systems don't always behave in a stable way; normally they evolve in an unstable or chaotic way. Furthermore, when a situation includes a great deal of different and heterogeneous variables, technical prediction becomes complex and unaffordable.

Anticipatory techniques [2] try to avoid the problem of using technical prediction in unstable systems by using the opinion of a group of human experts. The expert's opinion embodies relations among events or variables based on his/her personal experience; so complex relationships among heterogeneous events are mentally treated as a whole.

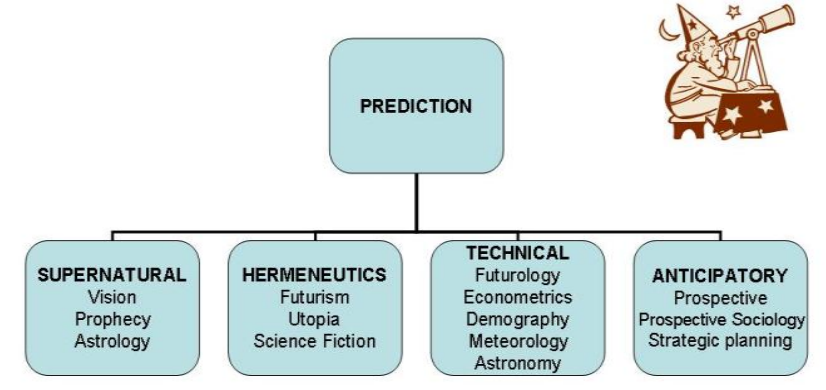

Figure 1. Ways to foresee the future

Technical prediction techniques are efficient in the creation of future scenarios based on stable dynamic systems in which tendencies of historical data are applied. However, inside the field of security it is hard to meet a stable dynamic system which generates scenarios based on predictable guidelines. The collapse of transports, economic crisis, natural disasters and terrorist attacks are just a few of many examples of scenarios of crisis which are difficult to estimate with techniques based upon technical prediction. Normally, the 
scenarios of crisis are created due to an accumulation of events that would otherwise be ineffective in isolation; however when occurring together they create an unsustainable and critical scenario.

In our every day lives there are many events, from domestic economy, incidence of criminality, social integration, to radical terrorist attacks. All these events belong to a specific scenario in which we are involved. We can study the future as the development of past and present events through the time.

From a conceptual point of view, our research is going to be developed under Prospective proceedings (instead of technical prediction). The final aim is to develop a technology which is able to identify and alert us to the generation of possible social scenarios of risk or crisis.

\section{BUILDING THE CRYSTAL BALL}

In this section, we illustrate a new approach for prospecting the future based on a Multi-Agent System [1] [7]. The objective consists of the construction of a model that faces the problem of modelling future scenarios from a different perspective from the classical statistical prospective methods. We use possibilities graded by linguistic tags instead of probabilities, we take a different track towards the problem compared to classical methods.

We have followed the MECIMPLAN [5] methodology to construct a software prototype that help us obtain results. This methodology describes the different steps and procedures to construct a MAS-oriented software prototype in this kind of domain. Nevertheless, MECIMPLAN can be used to develop intelligent systems, in both strategic [4] or tactical [3] planning.

\section{A. Methodology steps}

A methodology [5] that permits us to solve a wide range of planning problems is used in this section. In general, we can assume that the way in which we apply the method depends on a thorough analysis of the results obtained at each step. In certain circumstances, it is necessary to go back to previous states if the desired results are not obtained at a specific step.

To clarify the development of the software that supports our research we illustrate two specific phases of the methodology: Selection of agents and Model building.

\section{B. Selection of Agents}

We have used a neuro-fuzzy network [9] [14] aimed at reproducing human knowledge and experience in order to create a scenario by studying the influence among events. Thus, we talk about possibilities instead of probabilities and avoid using complex probabilistic techniques which are in most cases unclear for the human experts group.

We have implemented an intelligent search to make the sensitive analysis of variables (events) that can help us to arrive at an ideal scenario.

\section{Model Building}

We have built two agents in the MAS-oriented model: the Classifier agent and the Analyser agent. The first one will obtain the scenario after analyzing the proposed events. Each agent of the MAS has been developed to carry out a specific function; all of them are based on Artificial Intelligence procedures [11] [13].

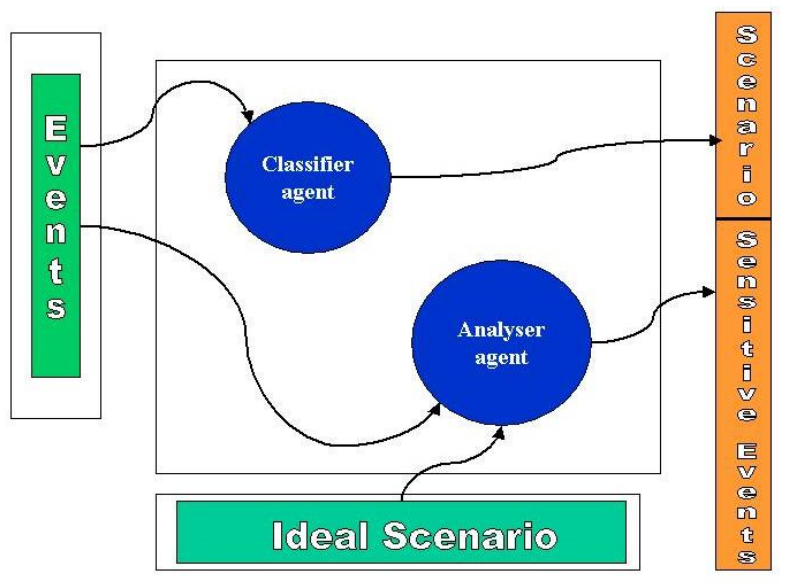

Figure 2. Conceptual Model

The knowledge extracted from the experts group will be used to train the Classifier Agent. Once the Classifier Agent has been trained, it can be used to generate new scenarios by presenting it with a set of events never used in the training phase. Thus, the knowledge of the experts group has been transferred to the Multi-agent System (MAS). It has been necessary to develop the classifier agent by means of fuzzy logic, since most of the times we express data in terms of adjectives. It is very common to define the relevance of the events or objectives in terms of linguistic tags. In this environment, Fuzzy Logic [16] provides a set of powerful tools.

The second agent is useful in determining which events can be influenced by us in order to arrive to the desired scenario. It is possible that the scenario doesn't match our expectations. In this case, the Analyser Agent is responsible for looking for the events which are to be influenced in order to get closer to an ideal scenario. We have used intelligent search as an Artificial Intelligence procedure to construct the Analyser Agent.

In Figure 2, we can observe the inputs to the model, the Agents we have designed to build the model, and the results we can obtain after its use. The model can be used for two purposes: to obtain a scenario as a result of the events, or to present an ideal scenario and look for the events that we have to influence in order to obtain or hinder such scenario.

In summary, the Classifier Agent receives the events and yields a scenario, while the Analyser Agent receives an ideal scenario and the original set of events and provides the list of events to be modified in order to obtain the ideal scenario. 


\section{WORKING WITH EXPERTS}

One of the key points of the process is to establish the method to elicit the information from each member of the experts group.

In order to extract the information from the experts group and with the intention of developing a MAS as a new solution, we suggest to follow the following steps:

- To select the experts group. The number of experts depends on the problem and the level of expertise they have. Usually a number of experts between 10 and 15 is accepted. If they actually are experts, to add more members to the group would produce redundant information.

- To generate a set of questionnaires comprising the whole field of different future scenarios.

- The experts will answer the questionnaires by using specific adjectives from the natural language instead of probabilities.

- Most likely each expert will have a particular view about the proposed scenario. Consequently, there will be different opinions inside the group of experts. From a technical point of view, the aim is to elicit the information from each expert and to develop a knowledge module able to give a response about future scenarios like the group of experts as a whole.

- Analysis of the most possible future scenario produced by the MAS as a result of the information given by the group of experts.

- Study and identification of the events that have a major influence in creating the scenario.

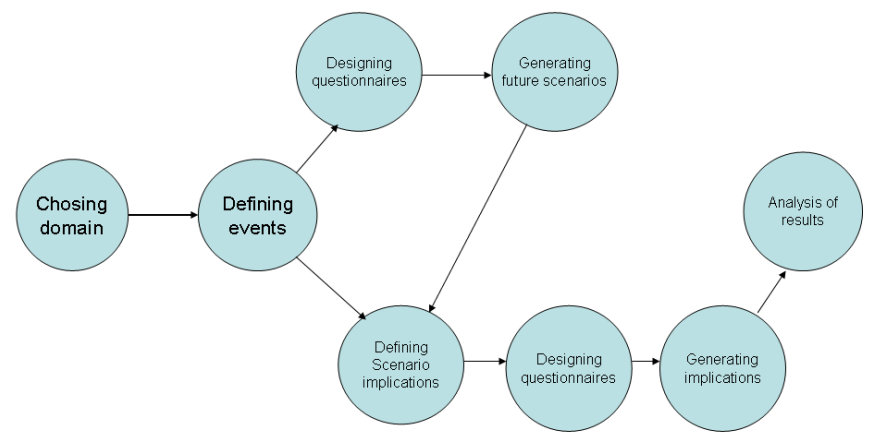

Figure 3. Map of processes

The above figure shows two branches. The upper branch describes the different processes that should be developed in order to generate future scenarios according to the opinions of a group of experts. In case we want to analyze the implications of the scenario generated in the upper branch or to study a specific scenario, we should proceed with the processes showed in the lower branch of the figure. We study the influence of external events not directly related to the scenario, on the possible scenario that is the objective of our study.

\section{V.A CASE STUDY FOR FORESEEING THE FUTURE OF THE COMMON POLICY OF SECURITY AND DEFENSE IN EUROPE}

In this section we present a work that is being developed by the Spanish Institute of Strategic Studies and Tecnalia.
The objective of this exercise of prospective is to foresee the future of the Common Policy and Defense in Security (CSDP) under the horizon of the year 2020.

\section{A. Defining the domain}

A group of analysts from the Spanish Institute of Strategic Studies chose the domain in which we should develop a prospective study. In this particular case, the objective consists of envisioning the future of the European Policy of Security and Defense in the year 2020.

\section{B. Defining events}

The same group of analysts with the assistance of a group of technical experts from Tecnalia defined the general events that are related to the domain. We chose the list of events paying special attention to their independence among them. A number of seven events were identified and are listed below:

Event 1: The public opinions of the member states press its governments for a major development of the CSDP.

Event 2: the structures are rationalized to promote the planning and execution of the missions of the CSDP, with an integrated employment of the civil and military capacities.

Event 3: a change takes place in the architecture of euro Atlantic security as consequence of a redefinition of the roles of the NATO and EU, and a change in the position of key actors as the USA and Russia.

Event 4: The ECSP (European Common Security Policy) develops of coherent form in accordance with the instruments foreseen in the Lisbon Treaty.

Event 5: The European Council decides for unanimity to implement a common European defense, in the terms established in the article 27.2 of the Lisbon Treaty.

Event 6: The Capabilities Headline Goals (military and civilian), which are established by the EU to substitute those of 2010, are accomplished.

Event 7: The EC constitutes a number of forces adequately trained and equipped, and ready to be used by flexibility in crisis management.

\section{Designing questionnaires}

Once the domain and the events were defined, we designed the questionnaires to be answered by the experts group. The number of questionnaires cannot be numerous and have to represent the whole range of possible scenarios.

The group of experts was chosen by the Institute of Strategic Studies. Fourteen experts on international policy were selected.

In order to facilitate the knowledge extraction process a website was developed for the experts to answer the questionnaires on-line (www.escenariosprospectiva.info). 


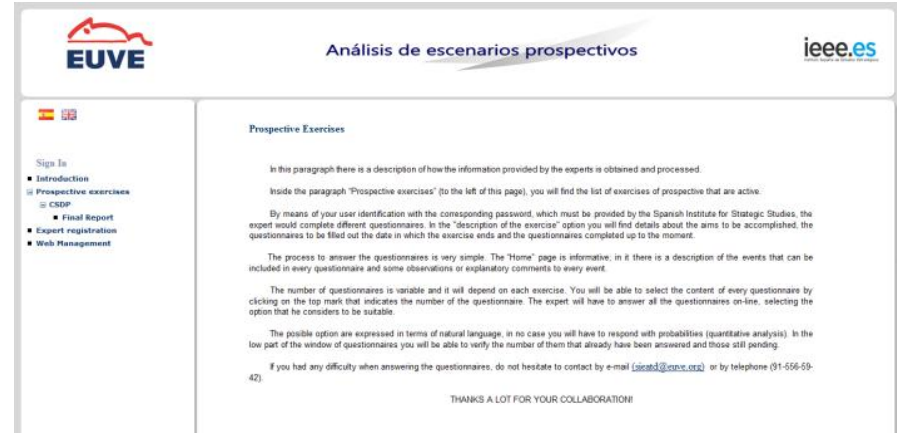

Figure 4. Eliciting information from experts

The number of events identified by the group of analysts is seven. Consequently, the number of possible scenarios is 128 . This number is the result of the different combinations of the events ( 2 raised to the power of 7). From the 128 possible scenarios a number of fifteen has been selected as the most representative of them. Each expert has to express his opinion about the existence of each scenario in terms of possibility as 'very high', 'high', 'medium', 'low' and 'very low'.

\section{Generating future scenarios}

After having studied the whole set of questionnaires, a number of fifty nine rules have been identified as the main elements to be treated as part of the multi-agent system. Namely, these fifty nine rules will be the core of the classifier agent. Currently, this task is still in process.

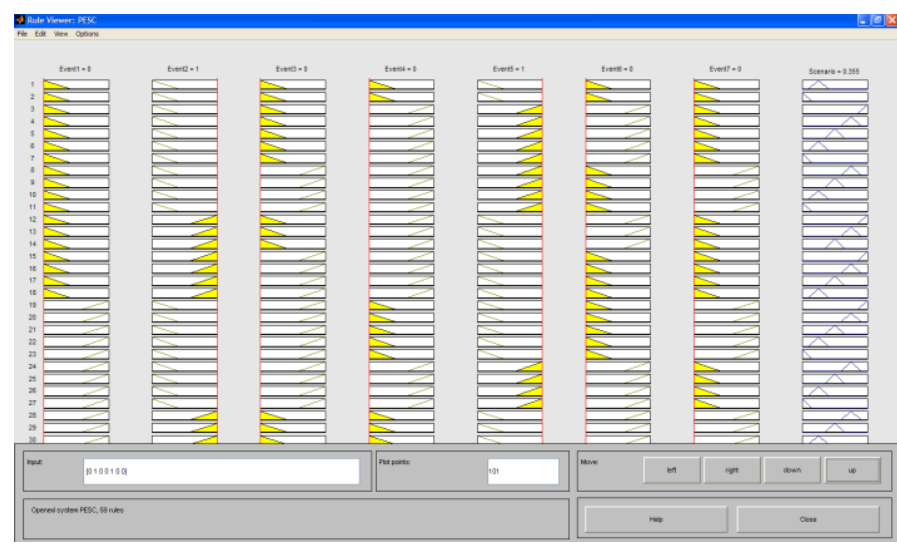

Figure 5. Rules generated by the experts

As describe in figure 2, the upper part of the map of processes is about to be completed. There are two tasks left to complete: the identification of the most possible scenario and the validation of the whole process.

Shortly, we are going to start with the lower part of the 'Map of processes' to study the implications of the most possible scenario and the analysis of factors to support the creation of the scenario or to prevent it from happening.

\section{PREVENTING CRISIS SCENARIOS IN SECURITY}

The technology presented in this paper is applicable to any field; especially in those that prevention plays an important role. That is the case of security in which when preventing a crisis or undesired scenario is crucial to avoid negative or fatal repercussions.

By treating adequately the opinion of human experts groups, it is possible to envision critical or undesired scenarios in the field of security. For example, this technology could be applied to detect emerging scenarios of terrorism in which different social events are involved.

Combining this technology with others like pattern recognition, tracing human groups' behaviour or analysis of tendencies most of the prevention activities in the field of security would be covered.

We are available to collaborate with any research group or governmental institution to validate the result of this research work for preventing future scenarios in the field of security.

\section{FUTURE WORKS}

The method and agent-based architecture permits not only to envision the most possible scenario but also to study its implications regarding other international and initially nonrelated scenarios.

At the present, we are tackling the study of the implications of the most possible scenario regarding the processes shown in the lower branch of figure 3 .

In order to validate the architecture and new approach showed in this article, in 2011 we are going to develop some prospective studies together with the Spanish Institute of Strategic Studies.

We are also planning to present a large scale European Project with the participation of four different Institutions of Strategic Studies from other European countries.

\section{VIII.CONCLUSION}

One of the most important advantages that this work can offer is the possibility of foreseeing future scenarios with the use of the agent-based technology.

Furthermore, by comparing our work with classical methods, we find the following advantages:

- The use of possibilities based on a natural use of linguistic tags instead of probabilities to define the possibility or intensity of events.

- The use of the concept of scenario implications expressed with global variables.

- A Sensitivity analysis of the events that should be modified in order to obtain an ideal scenario.

In this article, the applicability of the method is illustrated with a real case study that is still in process.

\section{ACKNOWLEDGMENTS}

This project has been partially funded by the Spanish Ministry of Science and Innovation under grant PID-5201002009-1, within the National plan of Scientific Research, Development and Technological Innovation (2008-2011). 


\section{REFERENCES}

[1] Aarsten, A., Brugali, D. y Vlad, C. (1996). Cooperating Among Autonomous Agents, Proceedings of the 4th International Conference on Control, Automation, Robotics, and Vision. Singapore

[2] Bas, E. (1999). Prospectiva. Cómo usar el pensamiento sobre el futuro. Ariel

[3] Castillo, J.M., Ossowski, S., Pastor, L., (2006); Planning Projectcs: A new approach through MECIMPLAN. Proc. of the IADIS Int. Conf. on e-Society. Dublin (Irland).

[4] Castillo, J.M., et al., (2006); Strategic Planning: A new approach through MECIMPLAN. Proc. of the IAT Int. Conf. Honk-Kong (China).

[5] Castillo, J.M. (2009); Una solución a la planificación de operaciones para la defensa basada en agentes inteligentes. Book. ISBN 978-849781-473-7

[6] Dalkey, N.C. (1975). Méthode Delphi. Dunod.

[7] Durfee, E. ; Cox, J. et ali. (2001). Integrating Multiagent Coordination with Reactive Plan Execution. Proceedings of the ACM Conference on Autonomous Agents (Agents-01), pages 149-150, June.

[8] Godet, M. (1993). De l'anticipation à l'action. Manuel de prospective et de stratégie. Dunod.

[9] Haykin, Simon, (1999). Neural networks. A comprehensive foundation. Prentice Hall

[10] Much, Richard et ali (1999). Intelligent Software Agents. Prentice Hall

[11] Nilsson, Nils J., (1998). Artificial Intelligence: A new synthesis. McGraw Hill.

[12] Riecken, D., (1994). An architecture of integrated agents. Communications of the ACM, 37(7):107-116

[13] Russell N., (2003). Artificial Intelligence: A modern approach. PrenticeHall.

[14] Sugeno, M., (1985). Industrial applications of fuzzy control. Elsevier Science Pub. Co

[15] Turoff, M.; (2009). The Past, Present and future of Delphi. FUTURA journal. Helsinki

[16] Zadeh, L.A., (1975). The concept of a linguistic variable and its application to approximate reasoning, Parts 1-3. Information Sciences.
José Miguel Castillo is the Director of the Area of Information Systems, training and decision making support in 'Tecnalia Research and Innovation'. In 2001 he earned his $\mathrm{PhD}$ in Telecommunications from the Universidad Politécnica in Madrid. The same year he was awarded with the 'General Fernández Chicarro' prize by the Spanish Ministry of Defence for his work on Operations Research.

$\mathrm{He}$ has a large experience leading projects in which simulation, artificial intelligence and project management are involved. He is Associate Professor at the Universidad Pontificia de Salamanca in Madrid. In 2007 he obtained his $\mathrm{PhD}$ in Computer Science from the Universidad Rey Juan Carlos in Madrid. In June 2007 he was awarded with the prize on Research by the Spanish Ministry of Defence. In the same year he was awarded with the prize on Research on the field of Security by the Directorate of Civil Protection and Emergencies (Spanish Ministry of Interior). (jmcastillo@euve.org). 